\title{
Comparison Effects of two Muscle Relaxant Strategies on Postoperative Pulmonary Complications in Transapical Transcatheter Aortic Valve Implantation: A Propensity Score-Matched Analysis
}

Hong Yu

Sichuan University West China Hospital

Yiding Zuo

Sichuan University West China Hospital

Zhao Xu

Sichuan University West China Hospital

Dailiang Zhao

Sichuan University West China Hospital

Jianming Yue

Sichuan University West China Hospital

Lulu Liu

Sichuan University West China Hospital

Yingqiang Guo

Sichuan University West China Hospital

Jiapeng Huang

University of Louisville

Xiaoqian Deng

Sichuan University West China Hospital

Peng Liang ( $\square$ liangpeng_world@foxmail.com )

West China Hospital of Sichuan university https://orcid.org/0000-0001-8485-1694

Research article

Keywords: Transcatheter aortic valve implantation, neuromuscular blockade, postoperative pulmonary complications, rocuronium/sugammadex, cisatracurium/neostigmine

Posted Date: October 28th, 2021

DOI: https://doi.org/10.21203/rs.3.rs-1006569/v1

License: (c) This work is licensed under a Creative Commons Attribution 4.0 International License. Read Full License 


\section{Abstract}

Background: Prior studies have reported conflicting results on the effect of sugammadex on postoperative pulmonary complications (PPCs) and research on this topic in transapical-transcatheter aortic valve implantation (TA-TAVI) was sparse. The current study aimed to investigate whether there were differences in the incidence of PPCs between two muscle relaxant strategies (rocuronium/sugammadex vs. cisatracurium/neostigmine) in patients undergoing TA-TAVI.

Methods: This retrospective observational study enrolled 245 adult patients underwent TA-TAVI between October 2018 and January 2021 . The patients were grouped according to the type of muscle relaxant strategies (115 with rocuronium/sugammadex in the R/S group and 130 with cisatracurium/neostigmine in the $\mathrm{C} / \mathrm{N}$ group, respectively). Pre- and intraoperative variables were managed by propensity score match (PSM) at a $1: 2$ ratio. PPCs (i.e., respiratory infection, pleural effusion, pneumothorax, atelectasis, respiratory failure, bronchospasm and aspiration pneumonitis) were evaluated from the radiological and laboratory findings.

Results: After PSM, 91 patients in the R/S group were selected and matched to 112 patients in the C/N group. Patients in the R/S group showed lower PPCs rate (45.1\% vs. $61.6 \%, \mathrm{p}=0.019)$ compared to the $\mathrm{C} / \mathrm{N}$ group. In addition, the R/S group showed significant shorter extubation time (7.2 \pm 6.2 vs. $10.3 \pm 8.2 \mathrm{~min}, p=0.003)$ and length of hospital stay ( $6.9 \pm 3.3$ vs. $8.0 \pm 4.0$ days, $p=0.034)$.

Conclusions: The rocuronium/sugammadex muscle relaxant strategy decreases the incidence of PPCs in patients undergoing TA-TAVI when compared to cisatracurium/neostigmine strategy.

Trial registration: ChiCTR, ChiCTR2100044269. Registered March 14, 2021-Prospectively registered, http://www.Chictr.org.cn

\section{Introduction}

Aortic valve disease is a common acquired valve diseases in adults(1), and aortic valve replacement (AVR) has been the only effective treatment which provides symptomatic relief and long-term survival(2). Recently, transcatheter aortic valve implantation (TAVI) has gained increasing acceptance as a safe and efficient alternative for patients with severe aortic valve disease(3-5).

Because of the minimally invasive nature of the TAVI approach, patients undergoing TAVI represent a suitable cohort for early recovery(6). However, transapical-TAVI (TA-TAVI) procedures need sufficiently profound neuromuscular blockade (NMB) during surgery which improves surgical conditions by inhibiting skeletal muscle movements. Therefore, postoperative residual NMB is one of the major hurdles for a faster recovery after surgery as it increases the risk of postoperative pulmonary complications (PPCs), such as hypoxemia(7), aspiration-induced pneumonia and reintubation(8). Furthermore, patients receiving TAVI often having underlying illnesses such as intrinsic lung disease (e.g., chronic obstructive pulmonary disease, COPD) and pulmonary dysfunction secondary to heart failure which increase their susceptibility to PPCs(9). Therefore, immediate restoration of patients' muscle strength at the end of TA-TAVI procedures are warranted for fast-track anesthesia to decrease respiratory problems.

Traditionally, acetylcholinesterase (AChE) inhibitors (e.g. neostigmine,) are commonly used to reverse NMB. However, the speed of neuromuscular function recovery is unpredictable(10). In addition, coadministration of choline antagonists is required to minimize muscarinic adverse effects (e.g. bradycardia, bronchoconstriction, hypersalivation)(11). Sugammadex, a modified $\gamma$-cyclodextrin, was designed for the reversal of NMB by encapsulating the steroidal NMB agents such as rocuronium and vecuronium(12). Sugammadex enables the reversal of deep NMB(13). Furthermore, sugammadex has no major adverse effects, including adverse cardiovascular effects, due to lack of endogenous targets(14).

Despite these advantages, the effects of sugammadex on PPCs are controversial(15-21). Especially, there are no evidence on sugammadex in high risk PPCs patients, including TA-TAVI cases. The aim of this study was to investigate the relationship between PPCs and two muscle relaxant strategies (rocuronium/sugammadex vs. cisatracurium/neostigmine) in TA-TAVI. Our primary outcome was a composite of in hospital PPCs and we hypothesized that there could be a significant reduction of PPCs in TA-TAVI patients who received rocuronium/sugammadex. We present the following article in accordance with the STROBE reporting checklist.

\section{Methods}

Ethical approval for this retrospective observational study (No.2019(591)) was provided by the Institutional Review Board (IRB) of West China Hospital of Sichuan University (Chairperson Prof Liu Lun-xu) on 18 December 2019. The requirement of informed consent was waived due to the retrospective nature of the analysis. The trial was registered at Chictr.org.cn (ChiCTR2100044269).

Data from adult patients who underwent TA-TAVI with endotracheal intubation and were successfully extubated in the operating room between August 2018 and January 2021 were collected retrospectively. Exclusion criteria included intubation before operating room arrival and extubation in the intensive care unit (ICU). In addition, patients with conversion to cardiopulmonary bypass (CPB) were excluded and those with incomplete or missing data were also excluded from this study. 
Since August 2018, our institution implemented enhanced recovery after surgery protocols in TA-TAVI patients and patients were routinely extubated in the operating room. Anesthesia was induced with 0.1 to $0.2 \mu \mathrm{g} / \mathrm{kg}$ of sufentanil, $1 \mathrm{mg}$ of midazolam, 1 to $2 \mathrm{mg} / \mathrm{kg}$ of propofol as necessary. Tracheal intubation was facilitated with either $0.6 \mathrm{mg} / \mathrm{kg}$ rocuronium or $0.2 \mathrm{mg} / \mathrm{kg}$ cisatracurium. Topical anesthesia of the glottis was performed by spraying with $3 \mathrm{ml}$ of $2 \%$ lidocaine before intubation. Anesthesia was maintained with sevoflurane or desflurane, 1.0 to 1.3 minimum alveolar concentration (MAC), $0.4 \mu \mathrm{g} / \mathrm{kg} / \mathrm{min}$ of dexmedetomidine, 0.1 to $0.3 \mu \mathrm{g} / \mathrm{kg} / \mathrm{min}$ of remifentanil, and $2 \mathrm{mg} / \mathrm{kg} / \mathrm{h}$ of lidocaine. The maintenance of effective concentrations was adjusted to achieve a target bispectral index (BIS) value of 40-60. Tropisetron $5 \mathrm{mg}$ was administrated intravenously for nausea or vomiting prophylaxis. Flurbiprofen $50 \mathrm{mg}$ was administrated before the end of surgery (unless contraindicated). $20 \mathrm{ml}$ of $0.5 \%$ ropivacaine was used for intercostal nerve blockade before skin closure. Dexmedetomidine was discontinued 40 minutes before the end of the surgery. Sevoflurane (if used) was discontinued and changed to desflurane at least 30 minutes before the end of the procedure. At the end of surgery, remifentanil and desflurane were discontinued. Fresh gas flow of $8 \mathrm{~L} / \mathrm{min} 100 \%$ oxygen were used to wash out inhalation anesthetics. Of note, train-of-four (TOF) ratio monitoring was not routinely used in our institution because of the limited resources, and anesthesiologists used one of two protocols for extubation. For the rocuronium/sugammadex protocol (the R/S group), a whole bottle of 200mg sugammadex (>2 mg/kg, the maximal body weigh was less than $100 \mathrm{~kg}$ ) was administrated immediately after skin closure for patients who received rocuronium. For the cisatracurium/neostigmine protocol (the $\mathrm{C} / \mathrm{N}$ group), neostigmine $0.04 \mathrm{mg} / \mathrm{kg}$ and atropine $0.02 \mathrm{mg} / \mathrm{kg}$ were administrated after spontaneous breathing recovery but not completely off the ventilator (insufficient tidal volume and/or frequency) for patients who received cisatracurium. For all cases, the tracheal extubation was carried out when patients were fully awake, responding to commands to open eyes, squeezing hands and lifting head for more than 5 seconds, and with adequate tidal volume and inspiratory force. The patient was then transferred to the ICU for further care.

Demographic and clinical data were collected from patients' electronic medical records, including preoperative comorbidities, anesthetic records, surgery-related data and chest computed tomography reports. All medical records were collected by Dai-liang Zhao and Jian-ming Yue who were blinded to the purpose of this study, and all of the researchers were blinded to the study data until after statistical outcomes were generated.

The primary outcome was occurrence of pulmonary complications during hospitalization according to European perioperative clinical outcome (EPCO) guidelines (respiratory infection, pleural effusion, pneumothorax, atelectasis, respiratory failure, bronchospasm and aspiration pneumonitis, methods in Supplemental Table S1)(22). We considered respiratory failure to be complications only when either noninvasive or invasive mechanical ventilation for oxygen therapy was initiated. All TA-TAVI patients routinely underwent their first chest computed tomography on the first or second postoperative day. Follow-up computed tomography was performed in patients with symptoms such as fever, coughing and sputum or in those with abnormalities on the first radiograph. We reviewed the radiological results until patients' discharge from hospital.

The secondary outcomes were: 1) extubation time: defined as from completion of surgery to extubation; 2) the length of stay (LOS) in ICU and 3) LOS in the hospital: defined as from surgery completion to discharge.

\section{Statistical analysis}

Propensity score matching was performed to minimize the risk of selection bias and confounder effects between the two groups. The patients were matched at a 1:2 ratio with a caliper of 0.2 . Propensity scores were calculated with a logistic regression analysis, and based on the covariates shown in table 1. An absolute standardized mean difference (SMD) less than $10 \%$ was considered to support the assumption of balance between the groups. $(23,24)$ The continuous variables were expressed as the mean with standard deviations or median with 25 th to 75 th percentiles. Categorical variables are shown as numbers (\%). Comparisons between two groups were done both in original cohort and propensity-matched cohort. Student's t-test was used for comparing continuous variables if the distribution was symmetric and Mann Whitney $U$ test if nonsymmetric, and chi-square or Fisher's exact test was used to compare categorical variables as appropriate. All data analyses were performed using SPSS version 25.0 software (SPSS Inc., IBM, Chicago, IL, USA) and a two-sided p-value of less than 0.05 was considered statistically significant.

\section{Results}

\section{Patient Characteristics}

In the initial cohort of 278 patients who underwent TA-TAVI between August 2018 and January 2021 at West China Hospital, we excluded 28 patients extubated in the ICU, 2 intubated already before operating room arrival and 3 converted to CPB. Finally, 245 patients were included in the analysis. Among them, 115 patients received rocuronium/sugammadex, and 130 patients received cisatracurium/neostigmine. Because these patients were not randomly assigned, there were statistically significant differences in NYHA functional class $(p=0.005)$ and previous stroke rate $(p=0.025)$ between the two groups.

After propensity score matching, a series of 91 patients receiving rocuronium/sugammadex matched to 112 patients receiving cisatracurium/neostigmine (Fig 1). The patients' characteristics and SMD values for the matched cohort are shown in Table 1. As expected, following matching, no significant difference between the two groups were detected regarding patient-related and surgery-related variables. 


\section{Primary outcomes}

After propensity score matching, there was statistically significant differences in the PPCs rate: $45.1 \%$ in the R/S group vs. $61.6 \%$ in the $\mathrm{C} / \mathrm{N}$ group $(p=0.028)$. There were no significant differences in the individual components of the primary outcome, including respiratory infection (11.0\% vs. $19.6 \%, p=0.092)$, atelectasis $(2.2 \%$ vs. $4.5 \%, p=0.379)$, pneumothorax $(6.6 \%$ vs. $7.1 \%, p=878)$ and respiratory failure $(26.4 \%$ vs. $32.1 \%, p=0.370)$. But there was statistically significant difference in the pleural effusion rate between the two groups (11.0\% in the R/S group vs. $19.6 \%$ in the $\mathrm{C} / \mathrm{N}$ group, $\mathrm{p}=0.003$ ). No aspiration pneumonitis nor bronchospasm occurred in either $\mathrm{R} / \mathrm{S}$ or $\mathrm{C} / \mathrm{N}$ group.

\section{Secondary outcomes}

After propensity score matching, the R/S group showed significant reductions in the extubation time $(7.2 \pm 6.2 \mathrm{vs}$. $10.3 \pm 8.2 \mathrm{~min}, \mathrm{p}=0.003)$ and length of hospital stay $(6.9 \pm 3.3$ vs. $8.0 \pm 4.0 \mathrm{~d}, \mathrm{p}=0.034)$ when compared to the $\mathrm{C} / \mathrm{N}$ group. However, the length of ICU stay $(24.5 \pm 17.1$ vs. $25.2 \pm 17.3$ days, $p=0.748$ ) were similar between two groups.

\section{Discussion}

This retrospective observational propensity score-matched study showed that the use of rocuronium/sugammadex compared to cisatracurium/neostigmine decreased the incidence of PPCs and pleural effusion in patients undergoing TA-TAVI. However, the incidence of respiratory infection, pneumothorax, atelectasis, respiratory failure, bronchospasm and aspiration pneumonitis did not differ significantly between the two groups. Of the secondary outcomes, the use of rocuronium/sugammadex was associated with shorter extubation time and length of hospital stay when compared to the use of cisatracurium/neostigmine.

TAVI is an efficient treatment for high-risk and intermediate risk surgical candidates with aortic valve disease, as well as those deemed to high risk to undergo open surgery $(25,26)$. Among current implanted prostheses available, only J-valve system is suitable for both severe aortic valve stenosis and regurgitation patients(27-29). However, the J-valve is still introduced through TA access which needs sufficient NMB to facilitate the surgical procedure. As a result, the use of nondepolarizing muscle relaxants could increase the risk of postoperative residual NMB and PPCs. Previous meta-analyses have shown that sugammadex reversed NMB more rapidly than neostigmine and was associated with fewer residual NMB rate (TOF ratio of less than 0.9$)(30,31)$. However, prior observational $(16,18,32)$ and randomized trials $(15,17,33)$ have reported conflicting results on the effect of sugammadex on PPCs.

Our study showed that rocuronium/sugammadex reduced the composite PPCs rate and pleural effusion rate. Unlike other relevant studies including non-cardiac patients $(15,17,34)$, the most common pulmonary complication observed in our study was pleural effusion rather than atelectasis. We considered all pleural effusion to be complications regardless the sides. Because unlike open heart surgery, the delivery catheter and implanted prostheses were inserted through the cardiac apex area on fifth intercostal space without opening the pleural cavity. Pleural effusion is a common complication after cardiac surgery as these TAVI patients are often complicated with heart failure, atrial fibrillation, peripheral vascular disease, receiving therapy with an anticoagulant or antiarrhythmic agent(35). From this, we enrolled the preoperative cardiac function parameters (i.e., NYHA functional class, LV ejection fraction, BNP, CK-MB, cTnT, intraoperative cardioversion or electric defibrillation event) in propensity score matching. After matching, the difference of pleural effusion between the two groups remained statistically significant. In addition, the residual NMB could also contribute to pleural effusion from incomplete recovery of respiratory muscular function(36). Sugammadex can quickly and efficiently re-establish normal muscle strength and cause less postoperative pleural effusion rate after TA-TAVI procedure. This finding was reinforced from a retrospective observational study by Han et al(17). They found that the postoperative pleural effusion rate was lower in patients receiving sugammadex when compared to patients receiving neostigmine, although they failed to found a significant difference of the incidence of PPCs between the groups(17). Furthermore, previous study showed that neostigmine can adversely affect neuromuscular function and impair muscle function (genioglossus muscle and diaphragm) which was associated with respiratory complications(37, 38).

There was a higher rate of NIV in our study (29.06\%) compared to other studies for non-cardiac surgeries (1.59\% to 12.16\%) (18-21). The high rate of COPD in our study may contribute to the higher NIV use. Some studies showed sugammadex was associated with less post-extubation desaturation and consequent NIV use $(18,20)$. However, in our study, the incidences of NIV $(26.4 \%$ vs. $31.1 \%)$ were similar between the two groups.

In our study, the extubation time was $7.2 \pm 6.2 \mathrm{~min}$ in the R/S group and $10.3 \pm 8.2 \mathrm{~min}$ in the $\mathrm{C} / \mathrm{N}$ group. Our study confirmed that rocuronium/sugammadex was superior to cisatracurium/neostigmine in reducing the extubation time. Lower residual NMB rate following the use of sugammadex $(15,30,31,33,39)$ may explain the faster extubation in the rocuronium/sugammadex group. Alternatively, this difference in extubation time could be explained by the fact that in the rocuronium/sugammadex protocol reversal agents were administered upon skin closure while the cisatracurium/neostigmine protocol required return of spontaneous breathing prior to dosing of reversal agents. Our finding was consistent with reports of two randomized studies including thoracic surgery with single lung ventilation $(33,40)$.

Another finding of this trial was that the LOS in hospital was 1.1 days shorter in the R/S group than the C/N group. This was consistent with reports of the association between the PPCs and prolonged hospital LOS $(41,42)$. However, several previous studies have not detected a reduction of hospital LOS with the use of sugammadex $(15,17,19,21,34,39,43,44)$. It might be explained by different study population between studies. 
We included patients with aortic valve disease who had poor clinical conditions from older age, more comorbidities and higher European system for cardiac risk evaluation (EuroSCORE) score when compared with other studies which included non-cardiac surgery patients(15, 17, 19, 21, 34, $39,43,44)$. As a result, the postoperative hospital LOS (7.5 days) in our study was longer than other studies (3.5 to 7.5 days) $(15,21,34,43-$

45) except one study including major abdominal surgery patients (12.5 days)(19).

Recapitulating the results of several studies, our study failed to detect a reduction in respiratory infection with the use of rocuronium/sugammadex $(15-17,19,34,40,45)$. However, the R/S group showed a significantly lower respiratory infection rate before matching $(8.7 \%$ vs $17.7 \%, p=0.04)$. Although there was no statistical significance after matching, the R/S group showed a relative $44 \%$ decrease of respiratory infection rate $(11.0 \%$ vs $19.6 \%, p=0.092)$ which was clinically significant. Actually, relevant studies involving non-cardiac surgeries reported an extremely low respiratory infection rate which ranged from $0.4 \%$ to $3.33 \%(15-17,19,34,40,45)$. We supposed that the results in the current and relevant studies might be explained by the insufficient power of the relatively low sample size to detect the difference in respiratory infection with lower event rates. Actually, in a large sample-sized observational study which included 45712 patients, a $47 \%$ reduced risk for respiratory infection (adjusted odds ratio, $0.53 ; 95 \% \mathrm{Cl}, 0.44$ to 0.62 ) was found in the sugammadex group compare to the neostigmine group(16).

This study has some limitations. First, this was a retrospective single center series of TA-TAVI. However, we used PSM based on almost all possible variables to control confounding factors. The second weakness is the lack of neuromuscular monitoring. Reversal with sugammadex in the absence of monitoring did not preclude residual neuromuscular block(46).

In conclusion, this propensity score match-based study showed that rocuronium/sugammadex decreased the incidence of PPCs in patients undergoing TA-TAVI. A sufficiently powered, prospective randomized study is warranted to confirm this effect size.

\section{Abbreviations}

AChE; acetylcholinesterase; AVR: aortic valve replacement; BIS: bispectral index; BMI: body mass index; CAD: coronary heart disease; C/N: cisatracurium/neostigmine; COPD: chronic obstructive pulmonary disease; CPB: cardiopulmonary bypass; CRF: chronic renal failure; EPCO: European perioperative clinical outcome; EuroSCORE: European system for cardiac risk evaluation; ICU: intensive care unit; LOS: length of stay; IRB: Institutional Review Board; LV: left ventricle; MAC: minimum alveolar concentration; MV: mitral valve; NIV: noninvasive ventilation; NMB: neuromuscular blockade; NYHA: New York Heart Association; PASO: peripheral arteriosclerosis obliterations; PPCs: postoperative pulmonary complications; PSM: propensity score match; R/S: rocuronium/sugammadex; SMD: standardized mean difference; TA-TAVI: transapicaltranscatheter aortic valve implantation; TOF: train-of-four.

\section{Declarations}

\section{Acknowledgements}

None declared.

\section{Authors' information (optional)}

Not applicable.

\section{Author contributions}

Conception and design: Peng Liang, Hong Yu and Xiaoqian Deng; Administrative support: Jiapeng Huang; Provision of study materials or patients: Lulu Liu and Yingqiang Guo; Collection and assembly of data: Yiding Zuo and Dailiang Zhao; Data analysis and interpretation: Xu Zhao and Jiangming Yue; Manuscript writing: All authors; Final approval of manuscript: All authors.

\section{Funding}

This work was supported by China National Natural Science Foundation (Grant No.81600918).

\section{Availability of data and materials}

The datasets used and/or analyzed during the current study are available from the corresponding author on reasonable request.

\section{Ethics approval and consent to participate}

Not applicable; IRB approved study.

\section{Consent for publication}


Not applicable.

\section{Competing interests}

The authors declare that they have no competing interests.

\section{References}

1. Thomas M, Schymik G, Walther T, Himbert D, Lefèvre T, Treede H, et al. Thirty-day results of the SAPIEN aortic Bioprosthesis European Outcome (SOURCE) Registry: A European registry of transcatheter aortic valve implantation using the Edwards SAPIEN valve. Circulation. 2010;122(1):62-9.

2. Birkmeyer JD, Stukel TA, Siewers AE, Goodney PP, Wennberg DE, Lucas FL. Surgeon volume and operative mortality in the United States. The New England journal of medicine. 2003;349(22):2117-27.

3. Baumgartner H, Falk V, Bax JJ, De Bonis M, Hamm C, Holm PJ, et al. 2017 ESC/EACTS Guidelines for the management of valvular heart disease. European heart journal. 2017;38(36):2739-91.

4. Waksman R, Rogers T, Torguson R, Gordon P, Ehsan A, Wilson SR, et al. Transcatheter Aortic Valve Replacement in Low-Risk Patients With Symptomatic Severe Aortic Stenosis. Journal of the American College of Cardiology. 2018;72(18):2095-105.

5. Mack MJ, Leon MB, Thourani VH, Makkar R, Kodali SK, Russo M, et al. Transcatheter Aortic-Valve Replacement with a Balloon-Expandable Valve in Low-Risk Patients. The New England journal of medicine. 2019;380(18):1695-705.

6. Papadopoulos N, El-Sayed Ahmad A, Thudt M, Fichtlscherer S, Meybohm P, Reyher C, et al. Successful fast track protocol implementation for patients undergoing transapical transcatheter aortic valve implantation. Journal of cardiothoracic surgery. 2016;11(1):55.

7. Sauer M, Stahn A, Soltesz S, Noeldge-Schomburg G, Mencke T. The influence of residual neuromuscular block on the incidence of critical respiratory events. A randomised, prospective, placebo-controlled trial. European journal of anaesthesiology. 2011;28(12):842-8.

8. Asai T, Isono S. Residual neuromuscular blockade after anesthesia: a possible cause of postoperative aspiration-induced pneumonia. Anesthesiology. 2014;120(2):260-2.

9. Weissman C. Pulmonary complications after cardiac surgery. Semin Cardiothorac Vasc Anesth. 2004;8(3):185-211.

10. Bartkowski RR. Incomplete reversal of pancuronium neuromuscular blockade by neostigmine, pyridostigmine, and edrophonium. Anesthesia and analgesia. 1987;66(7):594-8.

11. Keating GM. Sugammadex: A Review of Neuromuscular Blockade Reversal. Drugs. 2016;76(10):1041-52.

12. Gijsenbergh F, Ramael S, Houwing N, van lersel T. First human exposure of Org 25969, a novel agent to reverse the action of rocuronium bromide. Anesthesiology. 2005;103(4):695-703.

13. Madsen MV, Staehr-Rye AK, Gätke MR, Claudius C. Neuromuscular blockade for optimising surgical conditions during abdominal and gynaecological surgery: a systematic review. Acta anaesthesiologica Scandinavica. 2015;59(1):1-16.

14. Bom A, Bradley M, Cameron K, Clark JK, Van Egmond J, Feilden H, et al. A novel concept of reversing neuromuscular block: chemical encapsulation of rocuronium bromide by a cyclodextrin-based synthetic host. Angewandte Chemie (International ed in English). 2002;41(2):266-70.

15. Togioka BM, Yanez D, Aziz MF, Higgins JR, Tekkali P, Treggiari MM. Randomised controlled trial of sugammadex or neostigmine for reversal of neuromuscular block on the incidence of pulmonary complications in older adults undergoing prolonged surgery. British journal of anaesthesia. 2020;124(5):553-61.

16. Kheterpal S, Vaughn MT, Dubovoy TZ, Shah NJ, Bash LD, Colquhoun DA, et al. Sugammadex versus Neostigmine for Reversal of Neuromuscular Blockade and Postoperative Pulmonary Complications (STRONGER): A Multicenter Matched Cohort Analysis. Anesthesiology. 2020;132(6):1371-81.

17. Han J, Ryu JH, Koo BW, Nam SW, Cho SI, Oh AY. Effects of Sugammadex on Post-Operative Pulmonary Complications in Laparoscopic Gastrectomy: A Retrospective Cohort Study. Journal of clinical medicine. 2020;9(4).

18. Krause M, McWilliams SK, Bullard KJ, Mayes LM, Jameson LC, Mikulich-Gilbertson SK, et al. Neostigmine Versus Sugammadex for Reversal of Neuromuscular Blockade and Effects on Reintubation for Respiratory Failure or Newly Initiated Noninvasive Ventilation: An Interrupted Time Series Design. Anesthesia and analgesia. 2020;131(1):141-51.

19. Alday E, Muñoz M, Planas A, Mata E, Alvarez C. Effects of neuromuscular block reversal with sugammadex versus neostigmine on postoperative respiratory outcomes after major abdominal surgery: a randomized-controlled trial. Canadian journal of anaesthesia $=$ Journal canadien d'anesthesie. 2019;66(11):1328-37.

20. Ünal DY, Baran İ, Mutlu M, Ural G, Akkaya T, Özlü O. Comparison of Sugammadex versus Neostigmine Costs and Respiratory Complications in Patients with Obstructive Sleep Apnoea. Turkish journal of anaesthesiology and reanimation. 2015;43(6):387-95. 
21. Ezri T, Evron S, Petrov I, Schachter P, Berlovitz Y, Shimonov M. Residual Curarization and Postoperative Respiratory Complications Following Laparoscopic Sleeve Gastrectomy. The Effect of Reversal Agents: Sugammadex vs. Neostigmine. Journal of critical care medicine (Universitatea de Medicina si Farmacie din Targu-Mures). 2015;1(2):61-7.

22. Jammer I, Wickboldt N, Sander M, Smith A, Schultz MJ, Pelosi P, et al. Standards for definitions and use of outcome measures for clinical effectiveness research in perioperative medicine: European Perioperative Clinical Outcome (EPCO) definitions: a statement from the ESAESICM joint taskforce on perioperative outcome measures. Eur J Anaesthesiol. 2015;32(2):88-105.

23. PC A. An Introduction to Propensity Score Methods for Reducing the Effects of Confounding in Observational Studies. Multivariate behavioral research. 2011;46(3):399-424.

24. PC A. Propensity-score matching in the cardiovascular surgery literature from 2004 to 2006 : a systematic review and suggestions for improvement. The Journal of thoracic and cardiovascular surgery. 2007;134(5):1128-35.

25. Smith CR, Leon MB, Mack MJ, Miller DC, Moses JW, Svensson LG, et al. Transcatheter versus surgical aortic-valve replacement in high-risk patients. The New England journal of medicine. 2011;364(23):2187-98.

26. Leon MB, Smith CR, Mack MJ, Makkar RR, Svensson LG, Kodali SK, et al. Transcatheter or Surgical Aortic-Valve Replacement in IntermediateRisk Patients. The New England journal of medicine. 2016;374(17):1609-20.

27. Liu H, Yang Y, Wang W, Zhu D, Wei L, Guo K, et al. Transapical transcatheter aortic valve replacement for aortic regurgitation with a secondgeneration heart valve. J Thorac Cardiovasc Surg. 2018;156(1):106-16.

28. Zhu L, Guo Y, Wang W, Liu H, Yang Y, Wei L, et al. Transapical transcatheter aortic valve replacement with a novel transcatheter aortic valve replacement system in high-risk patients with severe aortic valve diseases. J Thorac Cardiovasc Surg. 2018;155(2):588-97.

29. Yu H, Zhao DL, Ye YC, Zheng JQ, Guo YQ, Zhu T, et al. Extubation in the Operating Room After Transapical Transcatheter Aortic Valve Implantation Safely Improves Time-Related Outcomes and Lowers Costs: A Propensity Score-Matched Analysis. J Cardiothorac Vasc Anesth. 2020.

30. Hristovska AM, Duch P, Allingstrup M, Afshari A. The comparative efficacy and safety of sugammadex and neostigmine in reversing neuromuscular blockade in adults. A Cochrane systematic review with meta-analysis and trial sequential analysis. Anaesthesia. 2018;73(5):631-41.

31. Hristovska AM, Duch P, Allingstrup M, Afshari A. Efficacy and safety of sugammadex versus neostigmine in reversing neuromuscular blockade in adults. Cochrane Database Syst Rev. 2017;8(8):Cd012763.

32. Li G, Freundlich RE, Gupta RK, Hayhurst CJ, Le CH, Martin BJ, et al. Postoperative Pulmonary Complications' Association with Sugammadex versus Neostigmine. Anesthesiology. 2021

33. Moon TS, Reznik S, Pak T, Jan K, Pruszynski J, Kim A, et al. Sugammadex versus neostigmine for reversal of rocuronium-induced neuromuscular blockade: A randomized, double-blinded study of thoracic surgical patients evaluating hypoxic episodes in the early postoperative period. J Clin Anesth. 2020;64:109804.

34. Yu J, Park JY, Lee Y, Hwang JH, Kim YK. Sugammadex versus neostigmine on postoperative pulmonary complications after robot-assisted laparoscopic prostatectomy: a propensity score-matched analysis. J Anesth. 2021.

35. Labidi M, Baillot R, Dionne B, Lacasse Y, Maltais F, Boulet LP. Pleural effusions following cardiac surgery: prevalence, risk factors, and clinical features. Chest. 2009;136(6):1604-11.

36. Kumar GV, Nair AP, Murthy HS, Jalaja KR, Ramachandra K, Parameshwara G. Residual neuromuscular blockade affects postoperative pulmonary function. Anesthesiology. 2012;117(6):1234-44.

37. Grosse-Sundrup M, Henneman JP, Sandberg WS, Bateman BT, Uribe JV, Nguyen NT, et al. Intermediate acting non-depolarizing neuromuscular blocking agents and risk of postoperative respiratory complications: prospective propensity score matched cohort study. Bmj. 2012;345:e6329.

38. Eikermann M, Fassbender P, Malhotra A, Takahashi M, Kubo S, Jordan AS, et al. Unwarranted administration of acetylcholinesterase inhibitors can impair genioglossus and diaphragm muscle function. Anesthesiology. 2007;107(4):621-9.

39. Motamed C, Bourgain JL. Comparison of the Time to Extubation and Length of Stay in the PACU after Sugammadex and Neostigmine Use in Two Types of Surgery: A Monocentric Retrospective Analysis. Journal of clinical medicine. 2021;10(4).

40. Çitil A, Alıcıkuş Tuncel Z, Yapici N, Kudsioğlu T, Aykac Z, Kavaklı A. Reversal of rocuronium induced neuromuscular blockade in lung resection surgery: a comparison of sugammadex and neostigmine. Journal of Cardio-Vascular-Thoracic Anaesthesia and Intensive Care Society. 2019.

41. Moore JA, Conway DH, Thomas N, Cummings D, Atkinson D. Impact of a peri-operative quality improvement programme on postoperative pulmonary complications. Anaesthesia. 2017;72(3):317-27.

42. Haines KJ, Skinner EH, Berney S. Association of postoperative pulmonary complications with delayed mobilisation following major abdominal surgery: an observational cohort study. Physiotherapy. 2013;99(2):119-25.

43. Evron S, Abelansky Y, Ezri T, Izakson A. Respiratory events with sugammadex vs. neostigmine following laparoscopic sleeve gastrectomy: a prospective pilot study assessing neuromuscular reversal strategies. Rom J Anaesth Intensive Care. 2017;24(2):111-4.

Page 7/12 
44. Llauradó S, Sabaté A, Ferreres E, Camprubí I, Cabrera A. Postoperative respiratory outcomes in laparoscopic bariatric surgery: comparison of a prospective group of patients whose neuromuscular blockade was reverted with sugammadex and a historical one reverted with neostigmine. Rev Esp Anestesiol Reanim. 2014;61(10):565-70.

45. Lee TY, Jeong SY, Jeong JH, Kim JH, Choi SR. Comparison of postoperative pulmonary complications between sugammadex and neostigmine in lung cancer patients undergoing video-assisted thoracoscopic lobectomy: a prospective double-blinded randomized trial. Anesth Pain Med (Seoul). 2021;16(1):60-7.

46. Kotake Y, Ochiai R, Suzuki T, Ogawa S, Takagi S, Ozaki M, et al. Reversal with sugammadex in the absence of monitoring did not preclude residual neuromuscular block. Anesthesia and analgesia. 2013;117(2):345-51.

\section{Tables}

Table 1 Baseline demographic and clinical characteristics for unmatched cohort and propensity-matched groups 
Original cohort $(n=245)$

Variables

Age (y)

Male gender (\%) 70(60.9)

$\left(\mathrm{kg} / \mathrm{m}^{2}\right)$

Smoking (\%)

$23.5 \pm 3.4$

NYHA functional 4 [3 to 4]

class

EuroSCORE II (\%) $\quad 10.53 \pm 7.77$

LV ejection

fraction (\%)

0.5

0.3-0.5

$55 \pm 13$

80(69.6)

32(27.8)

0.3

$3(2.6)$

Preop.
hemoglobin

CK-MB

cTnT

BNP

$\mathrm{Cr}$

GFR

Aortic morbidity

Stenosis

$0 / 1 / 2 / 3 / 4 / 5 / 6^{\dagger}$

Regurgitation

$0 / 1 / 2 / 3 / 4 / 5 / 6^{\dagger}$

$130 \pm 21$

$2.08 \pm 1.26$

$27.2 \pm 28.2$

$2810 \pm 4970$

$94 \pm 47$

$70.41 \pm 20.38$

71(61.7)

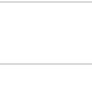

$44 / 7 / 1 / 4 / 1 / 56 / 2$

96(83.5)

19/18/6/10/19/39/4

\section{Both}

Preop. MV

regurgitation

\section{$0 / 1 / 2 / 3 / 4 / 5 / 6^{+}$}

Preop. Pulmonary

hypertension

$0 / 1 / 3 / 4 / 5^{\dagger} \quad 107 / 1 / 4 / 1 / 2$

Previous cardiac $17(14.8)$

surgery ${ }^{\ddagger}$

52(45.2)

53(46.1)

$62 / 27 / 7 / 11 / 6 / 1 / 1$

Comorbidities

Atrial fibrillation

Arterial
hyperten

hypertension

$\begin{array}{ll}\text { CAD } & 47(40.9)\end{array}$

Diabetes

18(15.7)
$\mathrm{C} / \mathrm{N}$ group

$(n=130)$

\begin{tabular}{l}
$70.5 \pm 6.1$ \\
\hline $77(59.2)$ \\
\hline $23.4 \pm 3.5$
\end{tabular}

32(24.6)

3 [3 to 4]

$9.90 \pm 6.36$

$57 \pm 12$

95(73.1)

31(23.8)

4(3.1)

$132 \pm 17$

$1.91 \pm 1.45$

$22.1 \pm 25.9$

$1855 \pm 3035$

$89 \pm 43$

$73.54 \pm 16.94$

77(59.2)

$53 / 3 / 0 / 7 / 8 / 55 / 0$

103(79.2)

$27 / 11 / 8 / 11 / 23 / 48 / 2$

50(38.5)

71(54.6)

$59 / 30 / 20 / 18 / 1 / 2 / 0$

7(5.4)

123/0/6/0/1

20(15.4)

16(12.3)

67(51.5)

$52(40.0)$

11(8.5)

Propensity-matched cohort $(n=203)$

$\begin{array}{lllll}\mathrm{P} & \mathrm{R} / \mathrm{S} \text { group } & \mathrm{C} / \mathrm{N} \text { group } & \mathrm{P} & \mathrm{SMD} \\ \text { value } & (\mathrm{n}=91) & \text { value } & \end{array}$

$0.110 \quad 71.1 \pm 6.0$

$71.0 \pm 5.9$

\begin{tabular}{lllll}
0.110 & $71.1 \pm 6.0$ & $71.0 \pm 5.9$ & 0.883 & 0.042 \\
0.794 & $53(58.2)$ & $66(58.9)$ & 0.921 & 0.022 \\
\hline 0.812 & $23.4 \pm 3.5$ & $23.4 \pm 3.6$ & 0.999 & 0.009 \\
0.383 & $22(24.2)$ & $29(25.9)$ & 0.779 & 0.048 \\
\hline 0.005 & $4[3$ to 4$]$ & $3[3$ to 4$]$ & 0.254 & 0.045
\end{tabular}

$\begin{array}{lllll}0.487 & 10.08 \pm 7.43 & 10.21 \pm 6.45 & 0.887 & 0.040 \\ 0.278 & 55 \pm 13 & 56 \pm 12 & 0.498 & 0.053\end{array}$

$\begin{array}{lllll}0.574 & 64(70.3) & 80(71.4) & 0.918 & - \\ & 25(27.5) & 28(25.0) & - \\ & 2(2.2) & 4(3.6) & -\end{array}$

$\begin{array}{lllll} & 2(2.2) & 4(3.6) & & - \\ 0.214 & 131 \pm 20 & 131 \pm 17 & 0.901 & 0.012\end{array}$

\begin{tabular}{lllll}
0.659 & $2.15 \pm 1.33$ & $1.85 \pm 1.21$ & 0.094 & 0.263 \\
\hline 0.146 & $26.1 \pm 28.9$ & $21.4 \pm 19.7$ & 0.172 & 0.190 \\
\hline 0.067 & $2577 \pm 5033$ & $1869 \pm 3041$ & 0.218 & 0.143 \\
\hline 0.368 & $86 \pm 29$ & $90 \pm 46$ & 0.475 & 0.065 \\
0192 & $73.23 \pm 17.85$ & $72.78 \pm 16.79$ & 0.855 & 0.047
\end{tabular}




\begin{tabular}{|c|c|c|c|c|c|c|c|}
\hline Previous stroke & $30(25.6)$ & $19(14.6)$ & 0.025 & $14(15.4)$ & 19(17.0) & 0.762 & 0.075 \\
\hline CRF & $10(8.7)$ & $7(5.4)$ & 0.309 & $4(4.4)$ & $7(6.3)$ & 0.562 & 0.058 \\
\hline COPD & $81(70.4)$ & $96(73.8)$ & 0.552 & 64(70.3) & 81(72.3) & 0.755 & 0.036 \\
\hline $\begin{array}{l}\text { Pulmonary } \\
\text { infection }\end{array}$ & 11(9.6) & $6(4.6)$ & 0.128 & $5(5.5)$ & $6(5.4)$ & 0.966 & 0.019 \\
\hline PASO & 61(53.0) & $69(53.1)$ & 0.996 & $45(49.5)$ & $56(50.0)$ & 0.938 & 0.044 \\
\hline \multicolumn{8}{|l|}{ Surgery-related } \\
\hline $\begin{array}{l}\text { Procedure status } \\
\text { (urgent/emergent) }\end{array}$ & $6(5.2)$ & $2(1.5)$ & 0.106 & $3(3.3)$ & $1(0.9)$ & 0.220 & 0.098 \\
\hline Surgical time, min & $81.7 \pm 23.2$ & $82.0 \pm 22.4$ & 0.923 & $82.1 \pm 24.2$ & $82.3 \pm 22.0$ & 0.965 & 0.003 \\
\hline \multicolumn{8}{|l|}{$\begin{array}{l}\text { Intraoperative } \\
\text { event }\end{array}$} \\
\hline Cardioversion & $5(4.3)$ & $2(1.5)$ & 0.188 & $3(3.3)$ & $2(1.8)$ & 0.490 & 0.080 \\
\hline $\begin{array}{c}\text { Electric } \\
\text { defibrillation }\end{array}$ & $7(6.1)$ & $2(1.5)$ & 0.059 & $5(5.5)$ & $1(0.9)$ & 0.054 & 0.206 \\
\hline
\end{tabular}

Categorical data are expressed as numbers (\%). Continuous data are expressed as mean \pm SD or median (interquartile range). †. $0=$ no, $1=$ mild, $2=$ mild to moderate, $3=$ moderate, $4=$ moderate to severe, $5=$ severe, $6=$ extremely severe; $\neq$. Previous cardiac surgery including pacemaker implantation, percutaneous transluminal coronary intervention and valve surgery. BMI: body mass index; CAD: coronary heart disease; $\mathrm{C} / \mathrm{N}$ : cisatracurium/neostigmine; COPD: chronic obstructive pulmonary disease; CRF: chronic renal failure (serum creatinine level $\geq 1.5 \mathrm{mg} / \mathrm{dl}$ in men or $\geq 1.3 \mathrm{mg} / \mathrm{dl}$ in women); EuroSCORE: European system for cardiac risk evaluation; LV: left ventricle; MV: mitral valve; NYHA: New York Heart Association; PASO: peripheral arteriosclerosis obliterations; R/S: rocuronium/sugammadex; SMD: standardized mean difference.

Table 2 Postoperative pulmonary complication rate and secondary outcomes in the original cohort and propensity-matched cohort 


\begin{tabular}{|c|c|c|c|c|c|c|c|c|}
\hline \multirow{3}{*}{ Variables } & \multicolumn{4}{|c|}{ Original cohort $(n=245)$} & \multicolumn{4}{|c|}{ Propensity-matched cohort $(n=203)$} \\
\hline & $\begin{array}{l}\mathrm{R} / \mathrm{S} \\
\text { group }\end{array}$ & $\begin{array}{l}\mathrm{C} / \mathrm{N} \\
\text { group }\end{array}$ & $\begin{array}{l}\text { Estimated } \\
\text { Difference }\end{array}$ & $\begin{array}{l}\mathrm{P} \\
\text { value }\end{array}$ & $\begin{array}{l}\mathrm{R} / \mathrm{S} \\
\text { group }\end{array}$ & $\begin{array}{l}\mathrm{C} / \mathrm{N} \\
\text { group }\end{array}$ & $\begin{array}{l}\text { Estimated } \\
\text { Difference }\end{array}$ & $\begin{array}{l}\mathrm{P} \\
\text { value }\end{array}$ \\
\hline & $(n=115)$ & $(n=130)$ & $(95 \% \mathrm{Cl})$ & & $(n=91)$ & $(n=112)$ & $(95 \% \mathrm{Cl})$ & \\
\hline Pulmonary complications & $54(47.0)$ & $81(62.3)$ & $\begin{array}{l}-15.3(-27.7 \text { to } \\
-3.0)\end{array}$ & 0.016 & $41(45.1)$ & $69(61.6)$ & $\begin{array}{l}-16.5(-30.1 \text { to } \\
-2.9)\end{array}$ & 0.019 \\
\hline Respiratory infection & $10(8.7)$ & 23(17.7) & $-9.0(-17.3$ to -0.3$)$ & 0.04 & $10(11.0)$ & 22(19.6) & $\begin{array}{l}-8.6(-18.4 \text { to } \\
1.2)\end{array}$ & 0.092 \\
\hline Pleural effusion & 18(15.7) & 43(33.1) & $\begin{array}{l}-17.4(-27.9 \text { to } \\
-6.9)\end{array}$ & 0.002 & 13(14.3) & $36(32.1)$ & $\begin{array}{l}-17.8(-28.1 \text { to } \\
-7.5)\end{array}$ & 0.003 \\
\hline Atelectasis & $2(1.7)$ & $6(4.6)$ & $-2.9(-7.2$ to 1.4$)$ & 0.206 & $2(2.2)$ & $5(4.5)$ & $-2.3(-6.8$ to 2.2$)$ & 0.379 \\
\hline Pneumothorax & $9(7.8)$ & $9(6.9)$ & $0.9(-5.7$ to 7.5$)$ & 0.787 & $6(6.6)$ & $8(7.1)$ & $-0.5(-6.8$ to 5.8$)$ & 0.878 \\
\hline Respiratory failure & $33(28.7)$ & 43(33.1) & $-4.4(-16.0$ to 7.2$)$ & & $24(26.4)$ & $36(32.1)$ & $\begin{array}{l}-5.7(-17.1 \text { to } \\
5.7)\end{array}$ & 0.370 \\
\hline Noninvasive ventilation & $33(28.7)$ & $42(32.3)$ & $-3.5(-15.0$ to 8.0$)$ & 0.540 & $24(26.4)$ & $35(31.3)$ & $\begin{array}{l}-4.9(-16.2 \text { to } \\
6.4)\end{array}$ & 0.447 \\
\hline Reintubation & $1(0.9)$ & $5(3.8)$ & $-2.9(-6.6$ to 0.8$)$ & 0.132 & $1(1.1)$ & $5(4.5)$ & $-3.4(-7.4$ to 0.6$)$ & 0.159 \\
\hline Aspiration pneumonitis & 0 & 0 & $\mathrm{n} / \mathrm{a}$ & $\mathrm{n} / \mathrm{a}$ & 0 & 0 & $\mathrm{n} / \mathrm{a}$ & $\mathrm{n} / \mathrm{a}$ \\
\hline Bronchospasm & 0 & 0 & $\mathrm{n} / \mathrm{a}$ & $\mathrm{n} / \mathrm{a}$ & 0 & 0 & $\mathrm{n} / \mathrm{a}$ & $\mathrm{n} / \mathrm{a}$ \\
\hline Extubation time (min) & $7.1 \pm 6.2$ & $10.1 \pm 8.0$ & $-3.0(-4.8$ to -1.2$)$ & 0.001 & $7.2 \pm 6.2$ & $10.3 \pm 8.2$ & $-3.1(-5.1$ to -1.1$)$ & 0.003 \\
\hline \multicolumn{9}{|l|}{ LOS } \\
\hline $\mathrm{ICU}, \mathrm{h}$ & $25.8 \pm 19.8$ & $25.0 \pm 16.3$ & $0.8(-3.8$ to 5.4$)$ & 0.734 & $24.5 \pm 17.1$ & $25.2 \pm 17.3$ & $-0.7(-5.5$ to 4.1$)$ & 0.748 \\
\hline $\begin{array}{l}\text { Surgery completion to } \\
\text { discharge, } d\end{array}$ & $6.8 \pm 3.0$ & $7.9 \pm 4.1$ & $-1.1(-2.0$ to -0.2$)$ & 0.025 & $6.9 \pm 3.3$ & $8.0 \pm 4.0$ & $-1.1(-2.1$ to -0.1$)$ & 0.034 \\
\hline
\end{tabular}

Categorical data are expressed as numbers (\%). Continuous data are expressed as mean $\pm \mathrm{SD}$. C/N: cisatracurium/neostigmine; LOS: length of stay; ICU: intensive care unit; R/S: rocuronium/sugammadex.

\section{Figures}



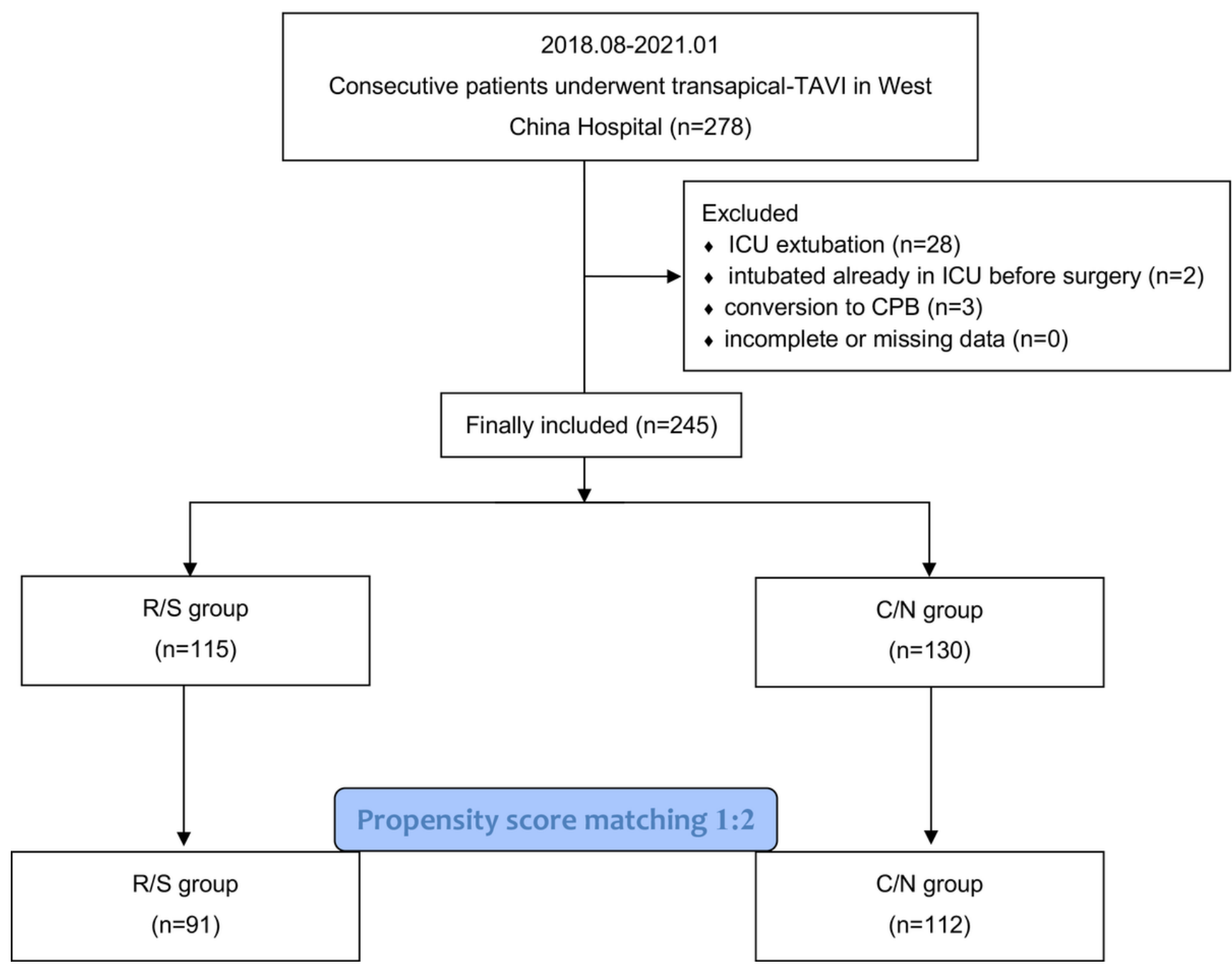

\section{Figure 1}

Flowchart displaying the identification of the matched pair groups. C/N: cisatracurium/neostigmine; CPB: cardiopulmonary bypass; ICU: Intensive care unit; R/S: rocuronium/sugammadex; TAVl: transcatheter aortic valve implantation.

\section{Supplementary Files}

This is a list of supplementary files associated with this preprint. Click to download.

- STROBEchecklistcohort.docx

- Table1SATM.docx 